\title{
BMJ Open Intestinal barrier function in obesity with or without metabolic syndrome: a systematic review protocol
}

\author{
Mariana Duarte Bona (D , ${ }^{1}$ Carlos Henrique de Medeiros Torres, ${ }^{2}$ \\ Severina Carla Vieira Cunha Lima, ${ }^{2,3}$ Aldo Angelo Moreira Lima, ${ }^{1}$ \\ Bruna Leal Lima Maciel $2,3,4$
}

To cite: Bona MD, Torres CHdM, Lima SCVC, et al. Intestinal barrier function in obesity with or without metabolic syndrome: a systematic review protocol. BMJ Open 2021;11:e043959. doi:10.1136/ bmjopen-2020-043959

- Prepublication history for this paper is available online. To view these files, please visit the journal online (http://dx.doi. org/10.1136/bmjopen-2020043959).

Received 20 August 2020 Accepted 19 April 2021

Check for updates

(c) Author(s) (or their employer(s)) 2021. Re-use permitted under CC BY-NC. No commercial re-use. See rights and permissions. Published by BMJ.

${ }^{1}$ Institute of Biomedicine for Brazilian Semiarid, Faculty of Medicine, Federal University of Ceara, Fortaleza, Brazil ${ }^{2}$ Department of Nutrition, Federal University of Rio Grande do Norte, Natal, Brazil

${ }^{3}$ Postgraduate Program in Nutrition, Federal University of Rio Grande do Norte, Natal, Brazil

${ }^{4}$ Postgraduate Program in Health Sciences, Federal University of Rio Grande do Norte, Natal, Brazil

Correspondence to Professor Bruna Leal Lima

Maciel;

brunalimamaciel@gmail.com

\section{ABSTRACT}

Introduction Intestinal barrier function is dependent on the structure and function of intestinal epithelial cells and paracellular pathway. The derangement of the intestinal barrier function can originate from conditions involving local and systemic chronic inflammation and metabolic diseases such as obesity and metabolic disorders. This study aims to describe a systematic review protocol investigating if obesity with or without metabolic syndrome is associated with an altered intestinal barrier function. Methods and analysis This protocol is guided by the Preferred Reporting Items for Systematic Reviews and Meta-Analyses Protocols. The databases to be searched are PubMed, Embase, Scopus, Science Direct and Web of Science. The systematic review will include original articles with adults and the elderly, who present obesity with or without metabolic syndrome, that address the intestinal barrier function. Two independent reviewers will perform study selection, data extraction and methodological quality assessment. Key information will be tabulated and a narrative synthesis will be conducted. The Grading of Recommendation, Assessment, Development and Evaluation framework will be used to assess the quality of evidence concerning the associations between intestinal barrier function and obesity with or without metabolic syndrome. The present protocol will assist in producing a systematic review that addresses if obesity with or without metabolic syndrome alters intestinal barrier function.

Ethics and dissemination No ethical statement will be required. The results will be disseminated through a peerreviewed publication and conference presentations. PROSPERO registration number CRD42020178658.

\section{INTRODUCTION}

The incidence of obesity and metabolic syndrome has risen significantly worldwide over the last decades and reached epidemic proportions affecting all ages and socioeconomic groups. ${ }^{12}$ Some evidence supports a causal pathway between diet, gut microbiota, intestinal barrier function and metabolic dysfunction. ${ }^{3-5}$ Most of this knowledge is based on animal studies, where the link between alterations in the gut microbiota and in intestinal barrier function was shown. ${ }^{67}$

\section{Strengths and limitations of this study}

- This study will focus on clinical research instead of the majority that focus on animal model researches.

- It will bring evidence of the most used in vivo tests to assess intestinal barrier function and integrity.

- In this study, individuals with obesity with or without metabolic syndrome will be included.

- The scarcity of researches with the elderly and the methodological quality of studies may be the main limitations of the study.

The intestinal barrier is a complex multilayer system, consisting of an external physical barrier and an inner functional immunological barrier. The interaction of these two barriers enables the maintenance of equilibrated intestinal barrier function. ${ }^{8}$ It prevents against loss of water and electrolytes and entry of antigens and microorganisms into the body while allowing the exchange of molecules between host and environment and the absorption of nutrients from the diet. $^{9}$

Many factors can alter intestinal barrier function such as gut microbiota modifications, mucus layer alterations and epithelial damage, resulting in translocation of luminal content to the inner layers of the intestinal epithelial cells. ${ }^{10}{ }^{11}$ Evidence obtained in animal models as well as in humans is accumulating, supporting a role of alterations of intestinal barrier function in many conditions, which include not only intestinal disorders such as malnutrition, diarrhoeal diseases, environmental enteric disease, inflammatory bowel disease, irritable bowel syndrome, hepatic fibrosis, inflammation, sepsis and pancreatitis, but also obesity and metabolic syndrome. ${ }^{12-15}$

Intestinal barrier function and integrity can be measured in different ways. The techniques used for this assessment vary depending on 
the setting (in vitro vs in vivo measurements), the species (human or animals), the marker molecules used (ions, carbohydrates of different sizes, macromolecules and antigens, bacterial products and bacteria), and the compartments used for measurement of the marker molecules (peripheral blood, portal vein blood or urine) ${ }^{16}{ }^{17}$ Each method is specific for a certain section of the gastrointestinal tract and measures different functional aspects of epithelial integrity of the intestine.

In vivo assessments of intestinal barrier absorption, damage and permeability in humans are currently possible by using intestinal barrier function biomarkers and assays. One of the most used assays is the oral lactulose:mannitol permeability test, a quantitative non-invasive test that directly measures the ability of two non-metabolised sugar molecules, lactulose and mannitol, to permeate the intestinal mucosa. ${ }^{18}$ Lactulose, a disaccharide, is absorbed through cell junctions or epithelial cell turnover or damage; while mannitol, a monosaccharide, is absorbed most across the epithelial cell membranes. ${ }^{19}$ Once absorbed, these sugars are excreted unmetabolised in the urine, and the sugar excretion is determined by chromatography. Elevated lactulose to mannitol ratio is an indicator of intestinal barrier dysfunction. ${ }^{11}$ Other sugar probes used to evaluate the intestinal barrier function include sucralose, rhamnose and sucrose, and these are also measured in the urine after an oral dose. ${ }^{20}$ The extent of sucrose absorption and subsequently excretion correlates with gastroduodenal permeation. ${ }^{3}$ Sucralose is resistant to bacterial utilisation in the colon and therefore has been used for measuring colonic permeability. ${ }^{21}$ Rhamnose is used as a marker for small bowel permeability. ${ }^{22}$ Sometimes, all these saccharide markers are used together to appraise pan-gastrointestinal permeability. ${ }^{3}$

The human protein zonulin is the main physiological modulator of tight junctions (TJs) in the intestinal epithelial layer that increases intestinal permeability in small intestine by inducing the opening of $\mathrm{TJ}$ and also participates in intestinal innate immunity. ${ }^{23}$ Circulating zonulin in serum is considered as a useful marker of intestinal barrier integrity and is measured using ELISA. ${ }^{24}$ In humans, it has been validated using lactulose/mannitol tests, being serum zonulin strongly correlated with the lactulose/mannitol ratio. ${ }^{25}$

Despite the test's immense potential, application in clinical research remains limited due to variations in the methodologies such as study population, sugar solution formulation and administration, urine collection time, assay method and sensitivity. ${ }^{26}$ These variations restrict the clinical sensibility and accuracy of the diagnostic tests of intestinal permeability, for example, the relationship between intestinal barrier function, integrity and inflammatory outcomes in diseases such as obesity and metabolic syndrome.

Animal models have shown that communication between the gut-adipose tissue and the gut-brain is essential for maintaining energy balance, and this communication is impaired during obesity and type 2 diabetes. ${ }^{27}$
In this context, metabolic endotoxemia, characterised by an increase in lipopolysaccharides in plasma, was identified as one of the main factors that leads to the development of metabolic inflammation and insulin resistance. Increasing evidence supports that the intestinal microflora is responsible for the development of a low-grade inflammation that generates dysfunctions in the intestinal barrier, increases its permeability and allows a consequent endotoxemia. ${ }^{28}$

Although these findings are well delineated for animal models, few studies in humans have been performed. ${ }^{29} \mathrm{~A}$ study compared two groups of women with and without obesity, assessing intestinal permeability by urinary lactulose/mannitol ratio. Although both sugars' urinary excretions were higher in women with obesity, a statistically significant difference in the lactulose/mannitol ratio was not found between the studied groups. Nevertheless, a higher lactulose/mannitol ratio was associated with higher homeostatic model assessment, insulin and low-density lipoprotein/high-density lipoprotein (HDL) concentrations, and lower HDL concentrations. ${ }^{30}$ Thus, the intestinal barrier function might be associated with obesity and metabolic syndrome.

This study aims to describe a systematic review protocol investigating if obesity with or without metabolic syndrome is associated with an altered intestinal barrier function. In addition, the systematic review will evaluate methodologies used in the studies regarding intestinal barrier function biomarkers and assay methods. This review protocol will address if obesity or obesity associated with metabolic syndrome alters intestinal barrier function and integrity.

\section{METHODS}

\section{Patient and public involvement}

No patients involved.

\section{Protocol and registration}

This protocol has been prepared according to the guidelines described in Preferred Reporting Items for Systematic Reviews and Meta-Analyses Protocols (PRISMA-P). ${ }^{31}$ A 27-item checklist was used to improve the quality of the systematic review data. The protocol was registered with the International Prospective Register of Systematic Reviews on 10 July 2020 and is available at: https:/ / www.crd.york.ac.uk/prospero/display_record.php?ID= CRD42020178658.

\section{Eligibility criteria}

Observational studies, published in scientific journals, will be included in the review. The guiding question in order to ensure the systematic search of available literature is: 'Is there alteration of intestinal barrier function in individuals with obesity with or without metabolic syndrome?' Thus, studies that have addressed as population individuals with obesity with or without metabolic syndrome assessing intestinal permeability as a variable will be included in the systematic review. 


\section{Inclusion criteria}

The review will include original articles studying adults and the elderly with obesity with or without metabolic syndrome (according to modified National Cholesterol Education Program criteria is the presence of three or more of the following components: (1) waist circumference more than $90 \mathrm{~cm}$ in men or $80 \mathrm{~cm}$ in women; (2) triacylglycerols equal to or more than $150 \mathrm{mg} / \mathrm{dL}$; (3) HDL cholesterol less than $40 \mathrm{mg} / \mathrm{dL}$ in men or $50 \mathrm{mg}$ / $\mathrm{dL}$ in women; (4) blood pressure equal to or more than 130/85 mg Hg; and (5) fasting glucose between 100 and $125 \mathrm{mg} / \mathrm{dL})),^{32}$ studies regarding the intestinal barrier function in this population and studies evaluating the intestinal epithelial cells integrity from individuals with obesity with or without metabolic syndrome.

All diagnostic tests for intestinal barrier function will be considered for the systematic review (urinary measurement of orally administrated sugar probe molecules and assays that use zonulin as a marker for intestinal permeability).

\section{Exclusion criteria}

Review articles, case reports, comments, editorials, letters to the editor, theses, conference proceedings, studies with animals or cell models, studies with children, studies with adults and/or the elderly that have other metabolic diseases, and studies that did not evaluate the intestinal barrier function.

\section{Information sources and literature search}

The search will be elaborated and implemented according to the PRISMA-P checklist as guidance. Search strategies will be developed based on keywords indexed in the Medical Subject Headings to identify the studies to be included in the systematic review. The descriptors used will be related to intestinal barrier function, obesity and metabolic syndrome, such as anthropometric data, biochemical analysis, intestinal permeability and integrity methodologies and assays. These descriptors will be accompanied by Boolean operators 'AND' and 'OR'.

Two reviewers will independently conduct sensitive search for eligible studies through the electronic databases PubMed Database, Embase Database, Cochrane Library, Scopus, Web of Science and Science Direct. In order to reflect the latest data, a search of the literature from the last 15 years (2006-2021) will be performed. Articles will be imported into Mendeley Reference Manager (V.1.19.4) and duplicates will be deleted. Initial screening of studies will be based on the information in their title, keywords and abstracts, following the eligibility criteria. When the reviewers disagree, the article will be re-evaluated and, if the disagreement persists, a third reviewer will make a final decision. Full-paper screening will be conducted by the same independent investigators. The references of the included articles will also be reviewed to identify those potentially eligible studies not found in the database search, considered as manual search.

\section{Data extraction}

For data extraction, two independent Microsoft Excel spreadsheets will be elaborated for two reviewers to summarise the data from the included studies. The following information will be extracted and entered in the spreadsheet: first author; year and language of publication; the country where the study was conducted; characteristics of the population (metabolic syndrome presence, age, gender, health conditions, total sample size, chronic diseases); methods to evaluate the intestinal barrier function; effects of obesity with or without metabolic syndrome in the intestinal barrier function; description of results and conclusions that are relevant to the overview; key findings and reported limitations.

\section{Methodological quality assessment}

Assessment of methodological quality and risk of bias in the studies with case-control design will be performed using the adapted Newcastle-Ottawa Scale, ${ }^{33}$ in which studies that receive at least five stars (maximum of eight) will be classified as good quality studies. Two independent reviewers will assess the methodological quality of eligible studies. These independent reviewers will score the selected studies, and a third reviewer will resolve any disagreement.

\section{Data analysis and synthesis}

The systematic review will describe the relevant information of the included studies. Key information on characteristics, methods, results and quality scores of included studies will be tabulated. Following this, a narrative synthesis will be conducted.

First, in the narrative review, the number of studies to be included in the synthesis will be reported and characteristics of each study will be described as well the location, kind and study population. Second, the narrative synthesis will report and discuss the methods used to evaluate the intestinal permeability and the relevant data. Also, the quality of the methods used will be discussed based on the related and observed study limitations. Finally, the observation of altered intestinal barrier function in obesity with or without metabolic syndrome will be explored and similarities and differences of findings will be reported.

The best-evidence synthesis will be guaranteed, and the risk of bias due to selective publication will be controlled by following the steps described above and assessing the quality of the evidence. The Grading of Recommendation, Assessment, Development and Evaluation (GRADE) framework will be used to assess the quality of evidence concerning the association between intestinal barrier function alteration in obesity with or without metabolic syndrome. GRADE ranks the evidence as high (when there is strong certainty that the association is close to the estimated); moderate (when there is moderate certainty in the estimated association); low (when certainty in association is limited); and very low (when certainty in the 
association estimate is very limited owing to a significant degree of uncertainty in the findings). ${ }^{34}$

\section{DISCUSSION}

Obesity has become a global epidemic and is a substantial threat to patients and healthcare systems because of related morbidity and costs. ${ }^{35}$ Metabolic and cardiovascular complications are a major obesity-associated burden, with critical roles for insulin resistance, type 2 diabetes and atherosclerosis. ${ }^{36}$ Given the increasing prevalence of obesity worldwide, it is necessary to identify individuals with or without metabolic syndrome as a clinical priority.

Evidence has proposed the potential role of the gut microbiota as a pathogenic factor affecting host metabolic balance and disorders. ${ }^{1}$ Gut microbiota seems to exert a great variety of functional properties impacting human physiology and pathology: modulation of host nutrition and energy harvest by the production of vitamins and fermentation of food components indigestible by the host; influence on intestinal epithelial homeostasis; intestinal barrier function; development of host immune system; protection against pathogens and drug metabolism. ${ }^{7}$

Animal models and some human studies are accumulating to support alterations of the intestinal barrier function in a vast array of conditions, which include obesity and metabolic syndrome. ${ }^{37}$ Given the importance of the intestinal barrier function and integrity, understanding what can disrupt it and cause the loss of function and integrity is necessary. Even though no final conclusions exist, it is more evident that besides nutrients acting as downregulators of TJs or as histone deacetylase inhibitors, also viral infections, toxins, hypoperfusion of the gut play a role. ${ }^{38}{ }^{39}$ Lifestyle factors such as living place, physical activity, dietary patterns and drug usage seem to play an important role as well, and they offer new approaches for improving gut barrier function. ${ }^{39}$

In this perspective, this systematic review will address studies that evaluated individuals with obesity with or without metabolic syndrome, focusing on the analysis of their intestinal barrier function. This review will also generate evidence for the use of different tests for the diagnosis of in vivo intestinal barrier function and integrity.

Acknowledgements The authors thank the Postgraduation Program in Medical Sciences of Federal University of Ceará and Postgraduation Program in Nutrition of Federal University of Rio Grande do Norte.

Contributors MDB, CHMT, SCVCL and BLLM conceived the idea, planned and designed the study protocol. MDB and CHMT wrote the first draft. SCVCL and BLLM planned the data extraction and statistical analysis. AAML and BLLM provided critical insights. All authors have approved and contributed to the final written manuscript

Funding This research was funded by the National Council for Scientific and Technological Development (award number: 406353/2018-2 CNPq).

Competing interests None declared.

Patient and public involvement Patients and/or the public were not involved in the design, or conduct, or reporting, or dissemination plans of this research.
Patient consent for publication Not required.

Provenance and peer review Not commissioned; externally peer reviewed.

Open access This is an open access article distributed in accordance with the Creative Commons Attribution Non Commercial (CC BY-NC 4.0) license, which permits others to distribute, remix, adapt, build upon this work non-commercially, and license their derivative works on different terms, provided the original work is properly cited, appropriate credit is given, any changes made indicated, and the use is non-commercial. See: http://creativecommons.org/licenses/by-nc/4.0/.

ORCID iD

Mariana Duarte Bona http://orcid.org/0000-0002-5856-2175

\section{REFERENCES}

1 Liu R, Hong J, Xu X. Gut microbiome and sérum metabolome alterations in obesity and after weight-loss intervention. Nature Medicine 2017;7:859-68.

2 Candi E, Tesauro M, Cardillo C, et al. Metabolic profiling of visceral adipose tissue from obese subjects with or without metabolic syndrome. Biochem J 2018;475:1019-35.

3 Khoshbin K, Camilleri M. Effects of dietary components on intestinal permeability in health and disease. Am J Physiol Gastrointest Liver Physiol 2020;319:G589-608.

4 Ridaura VK, Faith JJ, Rey FE, et al. Gut microbiota from twins discordant for obesity modulate metabolism in mice. Science 2013;341:1241214-22.

5 Cox AJ, West NP, Obesity CAW. Inflammation, and the gut microbiota. The Lancet 2015;3:207-15.

6 Cani PD, Possemiers S, Van de Wiele T, et al. Changes in gut microbiota control inflammation in obese mice through a mechanism involving GLP-2-driven improvement of gut permeability. Gut 2009;58:1091-103.

7 Tran CD, Grice DM, Wade B, et al. Gut permeability, its interaction with gut microflora and effects on metabolic health are mediated by the lymphatics system, liver and bile acid. Future Microbiol 2015;10:1339-53.

8 Scaldaferri F, Pizzoferrato M, Gerardi V. The gut barrier: new acquisitions and therapeutic approaches. Journal of Clinical Gastroenterology 2012;46:S12-17.

9 Brandtzaeg P. The gut as communicator between environment and host: immunological consequences. Eur J Pharmacol 2011;668:S16-32.

10 König J, Wells J, Cani PD, et al. Human intestinal barrier function in health and disease. Clin Trans/ Gastroenterol 2016;7:e196-13.

11 Leech B, Mclntyre E, Steel A, et al. Risk factors associated with intestinal permeability in an adult population: a systematic review. Int $J$ Clin Pract 2019;73:1-26.

12 Sánchez de Medina F, Romero-Calvo I, Mascaraque C, et al. Intestinal inflammation and mucosal barrier function. Inflamm Bowel Dis 2014;20:2394-404.

13 Kosek M, Guerrant RL, Kang G, et al. Assessment of environmental enteropathy in the MAL-ED cohort study: theoretical and analytic framework. Clin Infect Dis 2014;59:S239-47.

14 Kosek MN, Lee GO, Guerrant RL, et al. Age and sex normalization of intestinal permeability measures for the improved assessment of enteropathy in infancy and early childhood: results from the MAL-ED study. JPGN 2017;65:31-9.

15 Richard SA, McCormick BJJ, Murray-Kolb LE, et al. Enteric dysfunction and other factors associated with attained size at 5 years: MAL-ED birth cohort study findings. Am J Clin Nutr 2019;110:131-8.

16 Bischoff SC, Barbara G, Buurman W, et al. Intestinal permeability - a new target for disease prevention and therapy. BMC Gastroenterol 2014;14:1-25.

17 Galipeau HJ, Verdu EF. The complex task of measuring intestinal permeability in basic and clinical science. Neurogastroenterol. Motil. 2016;28:957-65.

18 Ordiz MI, Davitt C, Stephenson K, et al. EB 2017 Article: Interpretation of the lactulose:mannitol test in rural Malawian children at risk for perturbations in intestinal permeability. Exp Biol Med 2018;243:677-83.

19 Vojdani A. For the assessment of intestinal permeability, size matters. Altern Ther Health Med 2013;19:12-24.

20 Camilleri M, Lyle BJ, Madsen KL, et al. Role for diet in normal gut barrier function: developing guidance within the framework of food-labeling regulations. Am J Physiol Gastrointest Liver Physiol 2019;317:G17-39. 
21 Farhadi Aet al. Gas chromatografic method for detection of urinary sucralose: application to the assessment of intestinal permeability. $J$. Chromatogr B 2003;84:45-54.

22 Wilbrink J, Bernards N, Mujagic Z, et al. Intestinal barrier function in morbid obesity: results of a prospective study on the effect of sleeve gastrectomy. Int J Obes 2020;44:368-76.

23 Fasano A. Zonulin and its regulation of intestinal barrier function: the biological door to inflammation, autoimmunity, and cancer. Physiol Rev 2011;91:151-75.

24 Tripathi A, Lammers KM, Goldblum S, et al. Identification of human zonulin, a physiological modulator of tight junctions, as prehaptoglobin-2. Proc Natl Acad Sci U S A 2009;106:16799-804.

25 Sapone A, de Magistris L, Pietzak M, et al. Zonulin upregulation is associated with increased gut permeability in subjects with type 1 diabetes and their relatives. Diabetes 2006;55:1443-9.

26 Lee GO, McCormick BJJ, Seidman JC, et al. Infant nutritional status, feeding practices, enteropathogen exposure, socioeconomic status, and illness are associated with gut barrier function as assessed by the lactulose mannitol test in the MAL-ED birth cohort. Am J Trop Med Hyg 2017;97:281-90.

27 Geurts L, Neyrinck AM, Delzenne NM, et al. Gut microbiota controls adipose tissue expansion, gut barrier and glucose metabolism: novel insights into molecular targets and interventions using prebiotics. Benef Microbes 2014;5:3-17.

28 Cani PD, Osto M, Geurts L, et al. Involvement of gut microbiota in the development of low-grade inflammation and type 2 diabetes associated with obesity. Gut Microbes 2012;3:279-88.

29 Portune KJ. Gut microbiota, diet and obesity-related disorders: the good, the bad and the future challenges. Mol Nutr Food Res 2016;61:1-38.
30 Teixeira TFS, Collado MC, Ferreira CLLF, et al. Potential mechanisms for the emerging link between obesity and increased intestinal permeability. Nutrition Research 2012;32:637-47.

31 Moher D, Liberati A, Tetzlaff J, et al. Preferred reporting items for systematic reviews and meta-analyses: the PRISMA statement. PLoS Med 2009;6:e1000097-6.

32 Expert Panel on Detection, Evaluation, and Treatment of High Blood Cholesterol in Adults. Executive summary of the third report of the National cholesterol education program (NCEP) expert panel on detection, evaluation, and treatment of high blood cholesterol in adults (adult treatment panel III). JAMA 2001;285:2486-97.

33 The Newcastle-Ottawa quality assessment scale. Available: http:// www.ohri.ca/programs/clinical_epidemiology/nosgen.pdf [Accessed on 01 Mar 2021].

34 Guyatt GH, Oxman AD, Vist GE, et al. Grade: an emerging consensus on rating quality of evidence and strength of recommendations. $B M J$ 2008;336:924-6.

35 Blüher M. Obesity: global epidemiology and pathogenesis. Nat Rev Endocrinol 2019;15:288-98.

36 LT W, Shen YF, Hu L. Prevalence and associated factors of metabolic syndrome in adults: a population-based epidemilogical survey in Jiangi Province, China. BMC Public Health 2020;20:1-10.

37 Brignardello J, Morales P, Diaz E, et al. Pilot study: alterations of intestinal microbiota in obese humans are not associated with colonic inflammation or disturbances of barrier function. Aliment Pharmacol Ther 2010;32:1307-14.

38 Groschwitz KR, Hogan SP. Intestinal barrier function: molecular regulation and disease pathogenesis. J Allergy Clin Immunol 2009;124:3-20.

39 Bischoff SC. 'Gut health': a new objective in medicine? BMC Med 2011;9:1-14. 